\title{
Aggressive Blutdrucksenkung lohnt sich
}

Fragestellung: Führt eine aggressive antihypertensive Therapie zu einem Rückgang kardiovaskulärer Ereignisse?

Hintergrund: Die Hypertonie ist der wichtigste Risikofaktor für das Auftreten eines Schlaganfalls und der drittwichtigste Risikofaktor für den Myokardinfarkt. Eine Behandlung der arteriellen Hypertonie führt zu einer Reduktion von Schlaganfällen um $30-40 \%$ und zu einer Reduktion von Herzinfarkten um $15-25 \%$. Den größten Einfluss hat die antihypertensive Therapie auf die Entwicklung einer Herzinsuffizienz mit einem Risikoreduktion von bis zu $64 \%$. Umstritten ist nach wie vor, welches der ideale systolische Blutdruckwert bei der antihypertensiven Therapie ist.

Patienten und Methodik: In die Studie wurden 9.361 Patienten mit einem systolischen Blutdruck von $130 \mathrm{mmHg}$ und mehr aufgenommen, die kardiovaskuläre Risikofaktoren, aber keinen Diabetes hatten. Die Patienten wurden in einer Gruppe randomisiert, bei der angestrebt wurde, den systolischen Blutdruck unter $120 \mathrm{mmHg}$ einzustellen und in eine zweite Gruppe, bei denen der Blutdruck unter $140 \mathrm{mmHg}$ gesenkt wurde. Der kombinierte primäre Endpunkt umfasste Herzinfarkt, akutes Koronarsyndrom, Schlaganfall, Herzinsuffizienz und kardiovaskulären Tod.

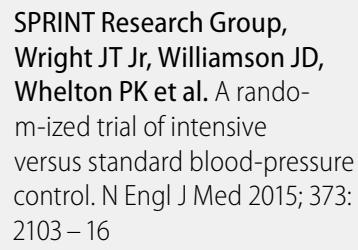

Ergebnisse: Die Patienten waren im Mittel 68 Jahre alt. Der initiale Blutdruck betrug $140 \mathrm{mmHg}$ systolisch und $78 \mathrm{mmHg}$ diastolisch. Etwa die Hälfte der Patienten nahm ein Statin oder Acetylsalicylsäure (ASS) ein. 13\% waren Raucher. Im Mittel nahmen die Patienten beim Studieneinschluss 1,8 verschiedene Antihypertensiva ein.

Ein Jahr nach Randomisierung betrug der mittlere systolische Blutdruck in der Intensivtherapiegruppe 121,4 $\mathrm{mmHg}$ und in der Standardtherapiegruppe 136,2 mmHg. Den primären Endpunkt erreichten 243 Patienten in der Intensivtherapiegruppe (5,2 \%, jährliche Ereignisrate von 1,65\%). In der Standardtherapiegruppe waren es 319 Patienten $(6,8 \%$, jährliche Ereignisrate $2,19 \%)$. Dies entspricht einer Hazard Ratio (HR) von 0,75, die mit einem p-Wert von 0,01 statistisch signifikant war.

Für die sekundären Endpunkte ergaben sich signifikante Unterschiede für Herzinsuffizienz, kardiovaskuläre Todesfälle und die Gesamtmortalität, wobei für die Endpunkte Herzinfarkt, akutes Koronarsyndrom und Schlaganfall keine signifikanten Unterschiede zu beobachten waren. Die Ergebnisse waren bei Patienten mit normaler Nierenfunktion deutlich ausgeprägter als bei Patienten mit eingeschränkter Nierenfunktion.

Subgruppenanalysen ergaben keine Unterschiede bezüglich Alter, Geschlecht, Rasse, vorbestehenden kardiovaskulären Erkrankungen und dem initialen Ausgangsblutdruck. Erwartungsgemäß traten mehr Nebenwirkungen in der Intensivtherapiegruppe auf. Statistisch signifikant häufiger waren Hypotonie, Synkopen, Elektrolytstörungen und Niereninsuffizienz. In der Intensivtherapiegruppe traten auch häufiger Hypokaliämien und Hyponatriämien auf.

Schlussfolgerungen: Bei Patienten mit hohem Risiko für kardiovaskuläre Ereignisse, aber ohne Diabetes mellitus, reduziert die Senkung des systolischen Blutdrucks unter $120 \mathrm{mmHg}$ verglichen mit unter $140 \mathrm{mmHg}$ das Risiko tödlicher und nicht tödlicher schwerwiegender kardiovaskulärer Ereignisse sowie die Mortalität. Dem steht eine erhöhte Rate an Nebenwirkungen gegenüber.

\section{- Kommentar von Hans-Christoph Diener Essen}

\section{Die Umsetzung in den klinischen Alltag wird schwierig sein}

Die SPRINT-Studie wurde angesichts der hochsignifikanten Ergebnisse vorzeitig abgebrochen. Die Ergebnisse geben Anlass die bisherigen Therapieziele von unter $140 \mathrm{~mm} \mathrm{Hg}$ systolisch bei Nichtdiabetikern und $130 \mathrm{~mm} \mathrm{Hg}$ bei Diabetikern zu überdenken. Die wesentlichen Ergebnisse beziehen sich auf die Mortalität und die Entwicklung einer Herzinsuffizienz. Die Studiendauer von 3,3 Jahren war wahrscheinlich zu kurz, um Effekte auf Herzinfarkt und Schlaganfall zu zeigen.

Die Umsetzung der Studie in die klinische Realität ist sicher schwierig, da eine aggressive antihypertensive Therapie mit nicht unerheblichen Nebenwirkungen einhergeht und sehr wahrscheinlich Probleme mit der Compliance bereitet. Die Ergebnisse sind im Moment auch nur auf Nichtdiabetiker zu übertragen, die bisher keinen Schlaganfall hatten. Welche idealen Grenzwerte für Patienten nach einem ischämischen
Insult oder einer zerebralen Blutung gelten, ist nach wie vor nicht geklärt.

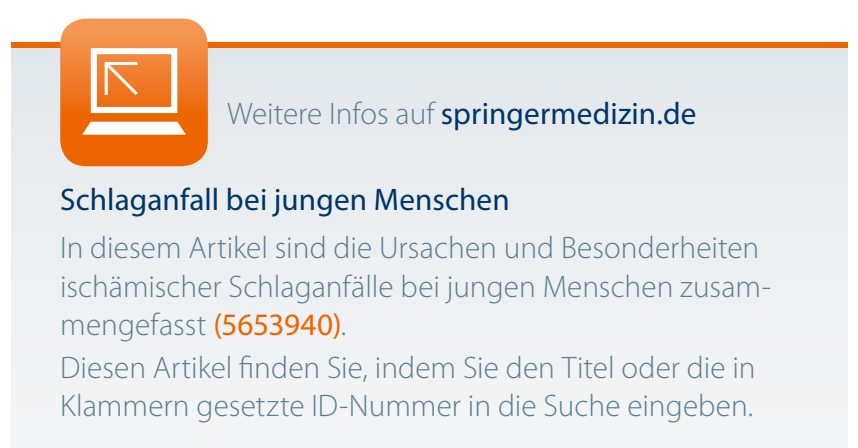

